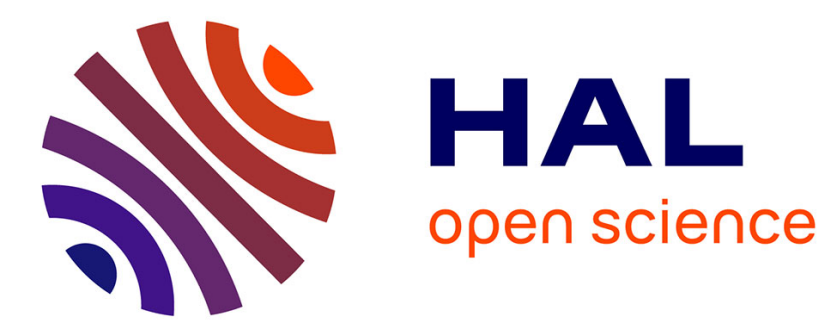

\title{
Shear-coupled grain-boundary migration dependence on normal strain/stress
}

Nicolas Combe, Frédéric Mompiou, Marc Legros

\section{To cite this version:}

Nicolas Combe, Frédéric Mompiou, Marc Legros. Shear-coupled grain-boundary migration dependence on normal strain/stress. Physical Review Materials, 2017, 1, pp.033605. 10.1103/PhysRevMaterials.1.033605 . hal-01726446

\section{HAL Id: hal-01726446 \\ https://hal.science/hal-01726446}

Submitted on 8 Mar 2018

HAL is a multi-disciplinary open access archive for the deposit and dissemination of scientific research documents, whether they are published or not. The documents may come from teaching and research institutions in France or abroad, or from public or private research centers.
L'archive ouverte pluridisciplinaire HAL, est destinée au dépôt et à la diffusion de documents scientifiques de niveau recherche, publiés ou non, émanant des établissements d'enseignement et de recherche français ou étrangers, des laboratoires publics ou privés. 


\title{
Shear-coupled grain boundary migration dependence on normal strain/stress
}

\author{
N. Combe, $,{ }^{1,2, \text { f F. Mompiou, }},{ }^{1,2}$ and M. Legros ${ }^{1,2}$ \\ ${ }^{1}$ Centre d'Elaboration de Matériaux et d'Etudes Structurales, CNRS UPR 8011, \\ 29 rue J. Marvig, BP 94347, 31055 Toulouse cedex 4, France \\ ${ }^{2}$ Université de Toulouse ; UPS ; F-31055 Toulouse, France
}

(Dated: June 23, 2017)

\begin{abstract}
In specific conditions, grain boundary (GB) migration occurs in polycrystalline materials as an alternative vector of plasticity compared to the usual dislocation activity. The shear-coupled GB migration, the expected most efficient GB based mechanism, couples the GB motion to an applied shear stress. Stresses on GB in polycrystalline materials have however seldom a unique pure shear component. This work investigates the influence of a normal strain on the shear coupled migration of a $\Sigma 13(320)[001] \mathrm{GB}$ in a copper bicrystal using atomistic simulations. We show that the yield shear stress inducing the GB migration strongly depends on the applied normal stress. Beyond, the application of a normal stress on this GB qualitatively modifies the GB migration: while the $\Sigma 13(320)[001]$ GB shear-couples following the $\langle 110\rangle$ migration mode without normal stress, we report the observation of the $\langle 010\rangle$ mode under a sufficiently high tensile normal stress. Using the Nudge Elastic Band method, we uncover the atomistic mechanism of this $\langle 010\rangle$ migration mode and energetically characterize it.
\end{abstract}

Plastic properties of metals are usually driven by the mobility of dislocations ${ }^{1}$. Because the dislocations motion is obstructed by the presence of grain boundaries (GB), the yield stress increases with the GB density as described by the Hall-Petch law 213. However, in nanocrystalline materials with grain sizes smaller than few hundreds nanometers, recent studies have shown that GB can themselves migrate and participate to the plasticity ${ }^{4}$ [9. Among the several identified GB migration mechanisms ${ }^{10}$, the shear coupled GB migration has focused a large attention because of its expected efficiency 11 . The displacement $m$ of the GB normally to its plane is accompanied by the relative in-plane displacement $d$ of the two grains forming the GBs. The coupling factor $\beta=\frac{d}{m}$ characterizes the amount of plastic shear produced by the GB migration.

Several previous studies have investigated various aspects of the shear coupled GB migration. For a given GB, geometric models theoretically predict different possible migration coupling modes, each characterized by a coupling factor $\frac{12}{14}$. Numerical studies relying on atomistic simulations and corroborating theoretical predictions have investigated GB-based mechanisms concentrating noticeably the efforts on the coupling factor or the mobility $\underline{1215}$ 20 . Interestingly, atomistic simulations have also recently succeeded in uncovering the role of disconnection ${ }^{2122}$ in the migration ${ }^{9123124}$. Remarkably, in all of the previous theoretical studies, the shear coupled GB migration is induced by applying a pure shear strain on the GB in absence of any other strain component on the GB.

Experimental quantitative studies on GB migration remain difficult: some, however have succeeded in measuring the coupling factor $\frac{417 / 8}{4}$ and have partially corroborated theoretical results. However, in these model experiments conducted on bicrystal, the GB plane normal forms a $45^{\circ}$ angle with respect to the straining axis, resulting in a maximum resolved shear stress/strain on the
GB but also in a normal tensile strain and stress $\frac{4725}{4}$. In experiments conducted on polycrystals, the local stress on a GB certainly has a shear component inducing its potential migration, but the meanwhile presence of a normal stress on the GB is highly probable, and surely even unavoidable.

We propose in this manuscript to theoretically address the influence of a non-shear component on the shear coupled GB migration mechanism. Though the effect of any component could, in principle be investigated, we here restrict our study on the effect of a normal strain. The effect of a normal load on the shear-coupled migration of $\Sigma 11(113)$ GBs in copper has been previously considered by molecular dynamics at finite temperature ${ }^{26}$ and shown to be non significant on the shear coupling. Below, we enrich this conclusion and give a deeper understanding of the effect of a normal stress.

Sect. I details the simulation methods and the system under study. In this section, we also precise the detailed boundary conditions under which the GB migration will be examined. Sect. III reports that the yield shear stress inducing the GB migration strongly depends on the applied normal stress. The application of a normal stress may even modify the operating coupling mode. Especially, in Sect. III we show that a coupling mode, the $\langle 010\rangle$ mode, that was theoretically predicted years agd 27 but had never been observed to our knowledge, can be activated by applying a tensile stress on the GB. The GB migration mechanism of this coupling mode is detailed. Finally, the activation energies of the GB migration are evaluated as a function of the applied shear and of the normal stress in Sect. IV.

\section{SIMULATION METHOD}

The shear-coupled migration of a symmetric tilt highangle $\Sigma 13(320)[001]$ GB is investigated in a $\mathrm{Cu}$ bicrys- 
tal model using the MD simulation package LAMMPS 28 with an embedded-atom method potential29. The choice of this peculiar GB and material is based on the several previous studies that have provided a good knowledge of the GB migration mechanisms i.e. mobility, structure and energetics ${ }^{9112 \mid 15}$.

Fig. 1 reports a sketch of the simulation cell defining the system under consideration. Two grains of a perfect fcc copper crystal are symmetrically disorientated relatively to each other by a angle $\theta=67.38^{\circ}$ around the [001] direction: a coincident site lattice tilt boundary $\Sigma 13(320)[001]$ results at the interface. Fig. 1b reports the GB equilibrium structure displaying characteristic structural units. Periodic boundary conditions are applied in
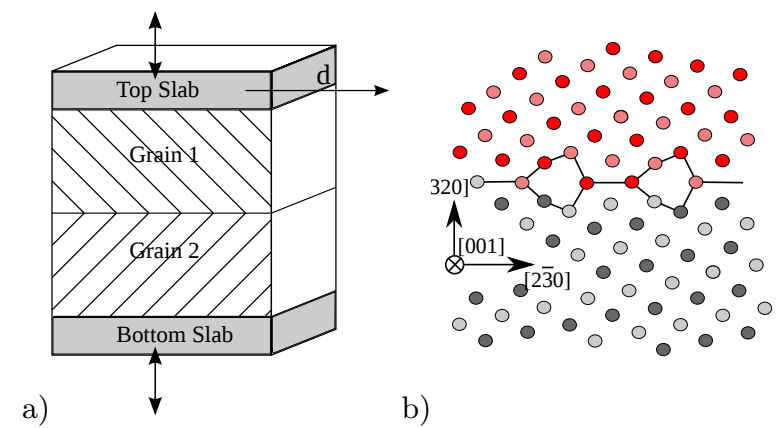

b)

FIG. 1. (color online) a) Sketch of the simulation model. b) Equilibrium structure of the $\Sigma 13(320)[001]$ GB projected along the [001] direction. Red (black) and pink (grey) atoms belongs to grain 1 (2). Red(black) and pink(grey) atoms do not belong the same (001) plane. For black and white printing, red, grey, and pink atoms appear as dark gray, gray, and light gray atoms.

the $[2 \overline{3} 0]$ and $[001]$ direction. In this manuscript, all crystal directions are given in the grain 1 lattice reference. Cell sizes are $12.06 \mathrm{~nm}, 6.5 \mathrm{~nm}, 2.88 \mathrm{~nm}$ along the [320], [250] and [001] directions, respectively. The cell contains about 19200 atoms. Strains/stresses are applied on the GB by displacing two $1.5 \mathrm{~nm}$ thick slabs at the bottom and top of the simulations cells. In these slabs, the relative atoms positions are frozen to the perfect lattices ones. The effects of the cell size on the results will not be reported in the present work, however the cell sizes and especially the one along the [320] direction have been carefully chosen in order to reveal unbiased GB migration mechanisms in Sect. III]

We have used the following procedure in order to apply the normal and shear strains/stresses on the GB. Tensile or compressive normal strains are first applied by displacing the slab along the [320] directions by increments of $0.022 \mathrm{~nm}$. After each slabs displacement (normally to the GB plane), the potential energy is minimized using a conjugate gradient algorithm The number of applied increments is chosen in order to reach the desired normal stress. After the application of this normal load, a shear strain is induced by the relative shear displacement $d$ of the two slabs along the $[2 \overline{3} 0]$ direction by increment of
$0.022 \mathrm{~nm}$. Again after each displacements, the potential energy is minimized. While the application of the shear stress follows a standard procedure widely used in the literature, we had to consider two extreme boundary conditions during the normal load.

- Clamped conditions (no in-plane strains): GB inplane strains are kept null during the normal load

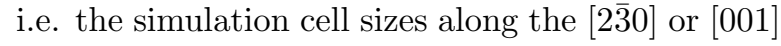
directions initially fixed to their equilibrium values are not relaxed. The application of the normal load thus induces stress components in the GB plane ${ }^{30}$.

- Free conditions (no in-plane stress): The cell is fully

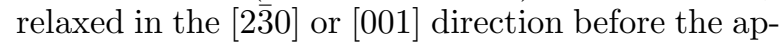
plication of the shear stress so that in-plane stress components are canceled at each step of the normal load.

While the clamped conditions would correspond to the application of a normal stress on a GB in a polycrystal where the presence of surrounding grains and/or triple junctions restrain biaxial relaxation in the GB plane, the free conditions would correspond to a fully relaxed material in the GB plane. Realistic conditions certainly stand in between these two extreme cases explaining our choice to consider both. While in principle, both conditions are relevant, only clamped conditions will be investigated in the following section for reasons mentioned below.

The system state is characterized by the relative shear displacement $d$ of the two slabs along the $[2 \overline{3} 0]$ direction and by the normal and shear stress components. Stresses tensors are computed using the virial expression 31 . Normal stresses ranging from $-5.5 \mathrm{GPa}$ (compressive) to $6 \mathrm{GPa}$ (tensile) have been investigated. While, for the clamped conditions, the grain boundary structure does not evolve over this range, for free conditions, we found that below a normal stress of $-2 \mathrm{GPa}$ and above $2 \mathrm{GPa}$, the grain boundary structure is modified presumably after a first order phase transition induced by the pressure. The modification of the GB migration due to a phase transition of the grain boundary structure induced by the temperature has already been observed ans studied ${ }^{32}$. In the following, we have chosen to focus on the migration of GBs preserving the same structure as the one displayed in Fig. 1p and thus on clamped boundary conditions. We will show that, though such study may appear limited, it brings significant new physics to the study of GB under complex loading conditions. The study of the GB structure and migration mechanism for free boundary conditions will no longer be considered here and will be the scope of a forthcoming publication.

\section{YIELD STRESS}

Fig 2a) reports the shear-stresses as a function of the relative slabs shear displacement $d$ for a normal stress of $\sigma_{N}=-1.97 \mathrm{GPa}$ and $\sigma_{N}=1.79 \mathrm{GPa}$ under clamped 
conditions. The normal stresses $\sigma_{N}=-1.97 \mathrm{GPa}$ and $\sigma_{N}=1.79 \mathrm{GPa}$ are chosen here as representative values among investigated normal stresses. Shearing the GB from $d=0$, the shear stress linearly increases as a function of the shear displacement $d$ with a slope depending on the applied normal stress in agreement with the linear elasticity theory 33 . A shear-stress drop signs a plastic event, namely the GB migration. The shear stress at which the migration occurs is called the yield shear stress in the following. A further increment of the shear displacement $d$ would increase the shear stress until the next GB migration, causing what has been called a stick-slip motion in MD simulations ${ }^{15}$. On the contrary, decreasing the shear displacement $d$ after the migration occurred will result in an elastic distortion of the GB in its final position. As seen in Fig. 2, the yield shear stress $\sigma_{y}$ inducing the GB migration is roughly 2.5 times higher in the presence of a tensile stress $\sigma_{N}=1.79 \mathrm{GPa}$ $\left(\sigma_{y}=2.3 \mathrm{GPa}\right)$ than for a compressive one $\sigma_{N}=-1.97$ $\mathrm{GPa}\left(\sigma_{y}=0.9 \mathrm{GPa}\right)$.
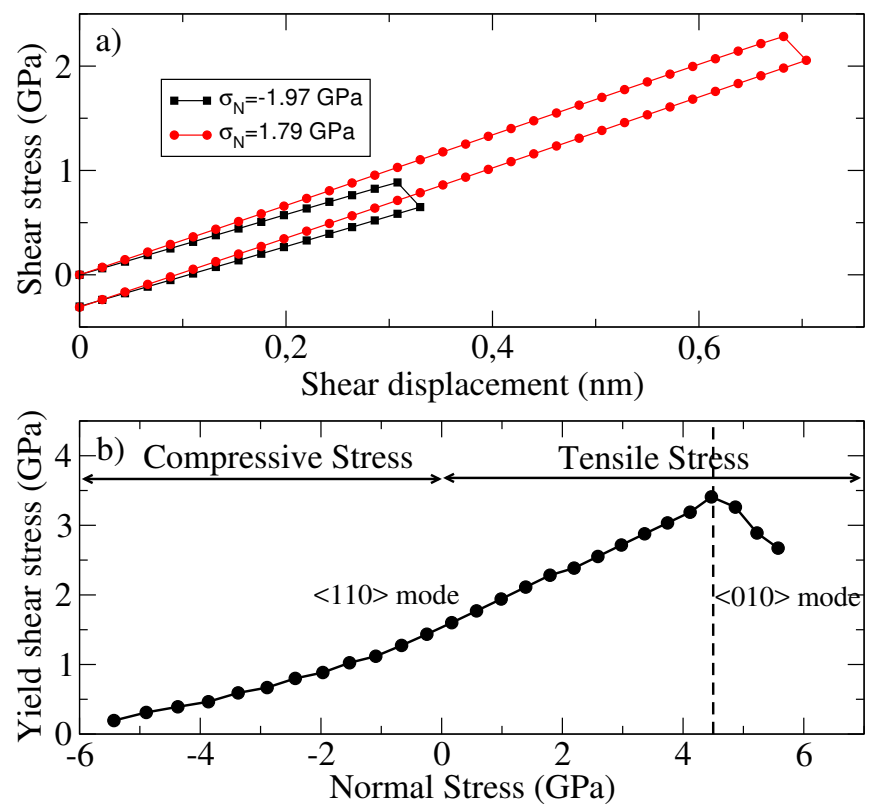

FIG. 2. (color online) a) Shear stress as a function of the shear displacement $d$ for applied tensile stresses $\sigma_{N}=1.79$ $\mathrm{GPa}$ and a compressive normal stress $\sigma_{N}=-1.97 \mathrm{GPa}$. b) Yield Shear stress as a function of the normal stress. Note that migration occurs at lower shear stress when the GB is under compression.

Fig. $2 \mathrm{p}$ reports the yield shear stress $\sigma_{y}$ as a function of the normal stress $\sigma_{N}$. The yield shear stress $\sigma_{y}$ is maximum for a normal stress of about $\sigma_{N}=4.5 \mathrm{GPa}$. For $\sigma_{N}<4.5 \mathrm{GPa}$, the yield stress of the shear-coupled GB migration increases with the normal stress: the measured slope $\frac{d \sigma_{y}}{d \sigma_{N}}$ is 0.4 at $\sigma_{N}=0$. Due to the magnitude of this slope, yield shear stresses $\sigma_{y}$ have the same order of magnitude as normal stresses. The dependence of the yield shear stress on the normal stress is thus highly relevant to understand experiments performed on bicrystal734. In- deed, in these later experiments, the GB plane normal is at $45^{\circ}$ angle with respect to the straining axis imposing the near equality of the shear and tensile normal stresses.

For $\sigma_{N}>4.5 \mathrm{GPa}$, interestingly, the yield shear stress decreases with the normal stress: as shown below, this change of qualitative behavior corresponds to a change of GB migration mechanism. Though in Fig. 2 $\mathrm{p}$, the range of investigated normal stresses is limited, the GB migrations with applied normal stresses below -6 GPa have also been tested (not shown). The yield shear stress $\sigma_{y}$ never cancels but presents a minimum at approximately $\sigma_{N}=-6 \mathrm{GPa}$ : below, $\sigma_{y}$ is a decreasing function of the normal stress. However, since below $\sigma_{N}=-6 \mathrm{GPa}$, the grain boundary structure is modified, this regime has not been investigated.

Instead, we focus below on the GB migration mechanisms occurring in the range $-5.5 \mathrm{GPa}$ (compressive) to $6 \mathrm{GPa}$ (tensile) in order to evidence the two regimes suggested by Fig. 2 .

\section{GB MIGRATIONS MECHANISMS}

In order to reveal the GB migration mechanisms, the reaction paths of the migration are calculated for different normal stresses. While at $T=0 \mathrm{~K}$, the migration occurs for an applied shear displacement corresponding to the yield shear stress, at finite temperature, the GB migration can be thermally activated for a smaller applied shear displacement $\frac{9124}{}$. In order to investigate the finite temperature migration, the reaction paths of the GB migration between configurations of the system before and after the GB migration are calculated for a given position of the slabs. Hence, each reaction path is characterized by the values of the normal stress and of the relative shear displacement. Investigated normal stresses are the same as the ones used to establish Fig. 2b. For a given normal stress, investigated shear displacements $d$ increase by increments of $0.022 \mathrm{~nm}$ from zero to the critical value inducing the GB migration (for $\sigma_{N}=-1.97$ $\mathrm{GPa}$ and $\sigma_{N}=1.79 \mathrm{GPa}$, these values correspond to the ones used to establish Fig. 2a). Reaction paths are calculated using the Nudge Elastic Band (NEB) method as a function of a Reaction Coordinate (RC), an indicator of the GB migration progress ${ }^{35}$ : the $\mathrm{RC}$ is here defined as the cumulative distance (normalized by the total $\mathrm{cu}-$ mulative distance) between adjacent replicas in the configuration space of dimension $3 \mathrm{~N}$ with $\mathrm{N}$ the number of atoms. For each NEB calculation, from 50 to $100 \mathrm{NEB}$ images have been used depending on the normal stress and shear displacement.

Fig. 3 reports the Minimum Energy Paths (MEP) as a function of the RC at a shear displacement $d=0.22$ $\mathrm{nm}$ for both applied normal stress $\sigma_{N}=1.79 \mathrm{GPa}$ and $\sigma_{N}=5.57 \mathrm{GPa}$. The value of this shear displacement and of the normal stresses $\sigma_{N}=1.79 \mathrm{GPa}$ and $\sigma_{N}=5.57$ $\mathrm{GPa}$ are chosen in order to report MEP that are both representative of the numerous calculated ones and of the 

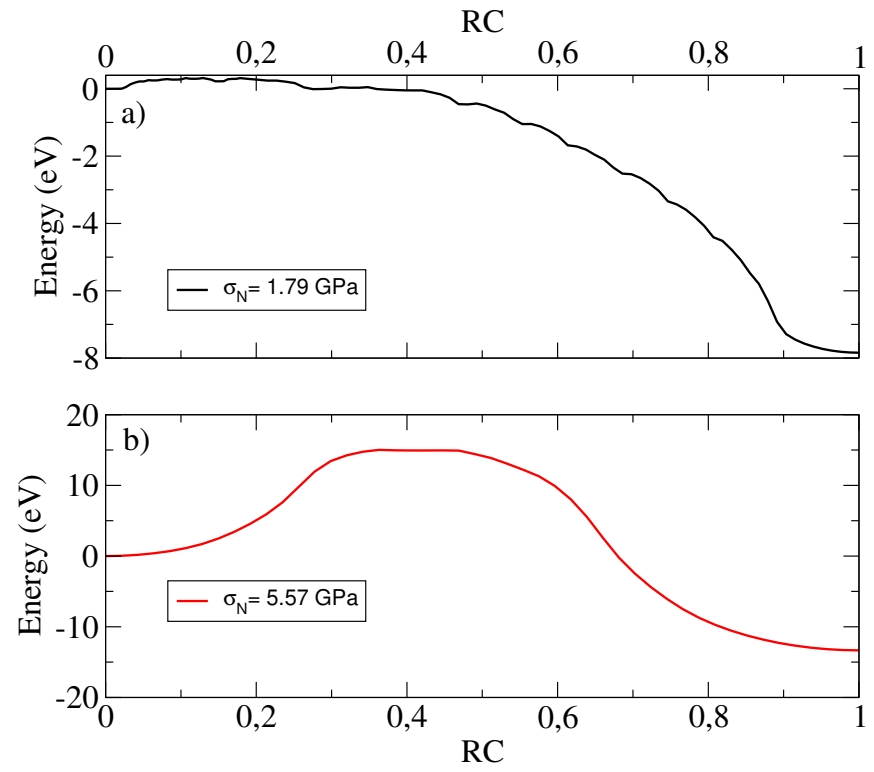

FIG. 3. (color online) MEP as a function of the RC for both tensile applied stress $\sigma_{N}=1.79$ (a) and $\sigma_{N}=5.57$ (b) at $d=0.22 \mathrm{~nm}$.

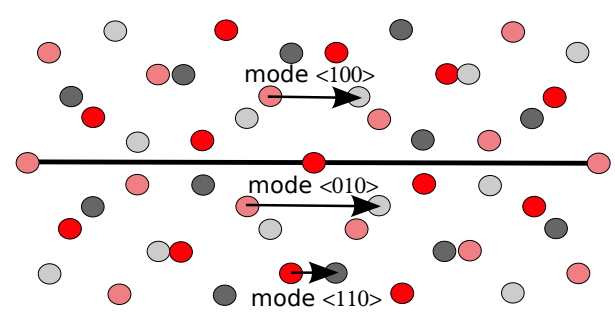

FIG. 4. (color online) Dichromatic pattern of the $\Sigma 13(320)[001]$ GB. Burgers vectors of the $\langle 110\rangle,\langle 010\rangle$ and $\langle 100\rangle$ migration modes are reported. The solid line materializes the GB plane. Same atom color code as in Fig. 1.

two regimes suggested by Fig. $2 \mathrm{p}$ i.e. below and above 4.5 $\mathrm{GPa}$. The two MEP displayed in Fig. 3 are qualitatively different. First, the activation energy of the GB migration for $\sigma_{N}=1.79 \mathrm{GPa}$ is roughly 50 times smaller than the one for $\sigma_{N}=5.57 \mathrm{GPa}$. The activation energy is defined here as the energy difference between the maximum energy along the MEP and the initial energy for $\mathrm{RC}=0$. Second, while the MEP for $\sigma_{N}=1.79 \mathrm{GPa}$ displays multiple metastable states, the MEP for $\sigma_{N}=5.57 \mathrm{GPa}$ is smooth and does not display any metastable state. These two main differences can be related to the GB migration mechanisms at the atomistic scale. The examination of the atomic configurations along both MEPs of Fig. 3 evidences the nucleation of two disconnections and their motion in opposite direction. These two disconnections have opposite Burgers vectors.

For $\sigma_{N}=1.79 \mathrm{GPa}$ and $d=0.22 \mathrm{~nm}$, performing a Burgers circuit analysis around one of these disconnections, the Burgers vector of the disconnection is $\frac{1}{13} a[320]$ (with $a$ the copper lattice parameter). From the measure of the normal GB migration distance (corresponding to the disconnection step height), we deduce the coupling factor $-\frac{2}{5}$ of this GB migration. Fig. 4 reports the dichromatic pattern of the $\Sigma 13(320)$ [001] GB and the Burgers vector of this disconnection. From the position of the Burgers vector in this dichromatic pattern, we identify the GB migration mode. Following the denomination of Cahn et al.12, this mode corresponds to the $\langle 110\rangle$ GB migration mode, a known mode that has been already observed and studied in the literature $27 / 36$. As suggested by Fig. $2 \mathrm{~b}$, a close examination of all nucleated disconnections along all calculated reaction path confirms the operation of the $\langle 110\rangle$ GB migration mode for $\sigma_{N}<4.5$ GPa. The detailed structure and motion mechanism of the $\langle 110\rangle$ mode disconnections have already been studied in Ref. 9 and will thus not be reported here again since we recover similar results. In this manuscript, the results concerning the mode $\langle 110\rangle$ will be limited to the calculations of the activation energies as a function of the shear displacement and of the normal stress in Sect. IV] a key element for the discussion of Sect. V.

For $\sigma_{N}=5.57 \mathrm{GPa}$ and $d=0.22 \mathrm{~nm}$, Fig. $5 \mathrm{a}$ and b respectively report the atomic configuration of the system along the MEP for $R C=0.49$ displaying two disconnections and the details of one of these disconnections. Performing a Burgers circuit analysis, the Burgers vector of this disconnection is $\frac{3}{13} a[320]$. From the measure of the disconnection step height, the coupling factor is $-\frac{1}{3}$. Fig. 4 reports the disconnection Burgers vector in the Dichromatic pattern. Following the denomination of Cahn et al ${ }^{12}$, this Burgers vector corresponds to the $\langle 010\rangle$ GB migration mode. While both $\langle 110\rangle$ and $\langle 100\rangle$ modes are the two known and observed modes during the shear coupling migration of tilt [001] GB depending on the orientation or of the disorientation angle ${ }^{\sqrt{12}}$, to our knowledge, this work is the first to propose some numerical conditions under which the $\langle 010\rangle$ GB migration mode is observed. In order to explicitly distinguish this mode from the $\langle 100\rangle$ one, the Burgers vector of the $\langle 100\rangle$ modes has also been reported in the Fig. 4. Examining all the nucleated disconnections along all calculated reaction path confirms the operation of the $\langle 010\rangle$ GB migration mode for $\sigma_{N}>4.5 \mathrm{GPa}$.

Hence, the change of the qualitative behavior of the yield shear stress as a function of the normal stress observed in Fig. $2 \mathrm{~b}$ corresponds to a change of GB migration mechanism from the $\langle 110\rangle$ to the $\langle 010\rangle$ mode.

In fig. $5 \mathrm{a}$ and $\mathrm{b}$, the large Burgers vector norm of the $\langle 010\rangle$ disconnections and the very high normal stress is responsible of the highly distorted structural units observed in the vicinity of the disconnections. The $\langle 010\rangle$ mode disconnection carries a Burgers vector with a very large norm $\frac{3}{\sqrt{13}} a$ corresponding to about $83 \%$ of the lattice parameter. This Burgers vector norm is three times larger than the one carried by the $\langle 110\rangle$ mode disconnection. In fig. $5 \mathrm{a}$ and $\mathrm{b}$, the solid line, drawn to highlight the structural units, has been constructed from the observation of the GB before and after the migration. The 


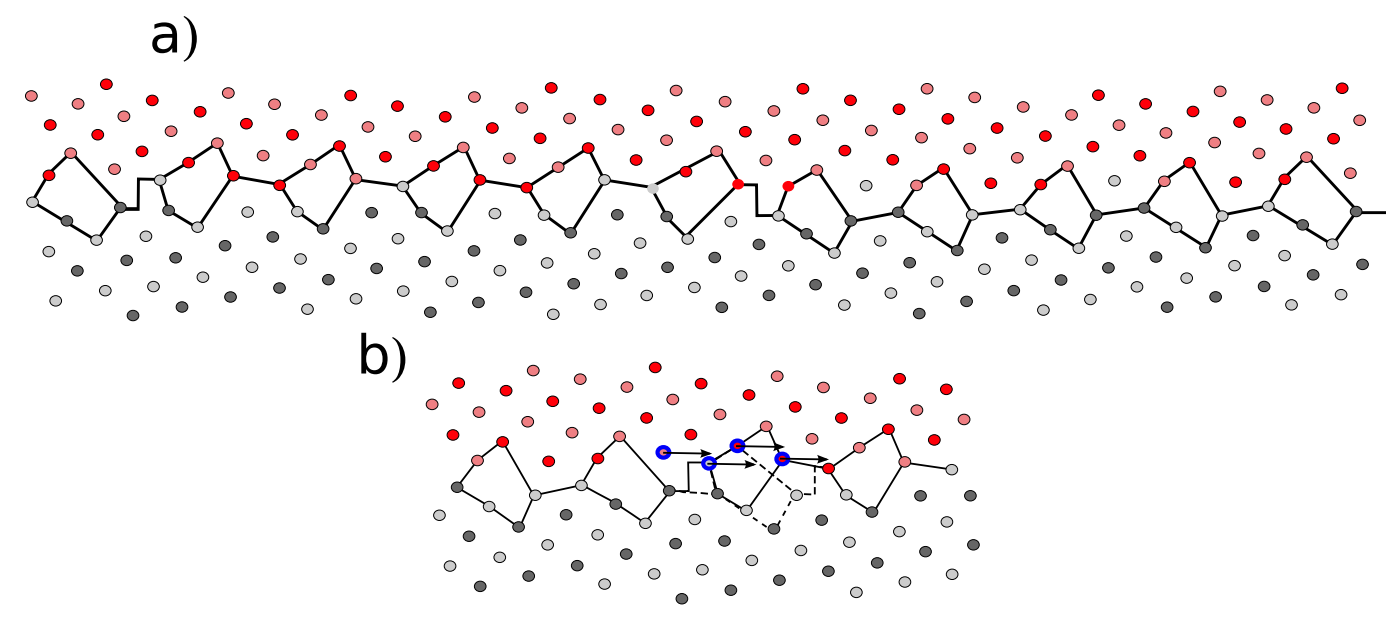

FIG. 5. (color online) a) Atomic configuration of the system along the MEP for $R C=0.49$ for $\sigma_{N}=5.57$ GPa for $R C=0.49$ at $d=0.22 \mathrm{~nm}$ projected along the [001] direction. b) Details of the atomic configuration of one disconnection. The solid line, a guide to the eye displays the GB position and reveals the structural units. A dashed line reports how this line will be modified by the disconnection motion (to the left). Same color code as Fig. 1.

disconnection motion is induced by atomic displacements corresponding to a shuffling mechanism, also observed in the case of $\langle 100\rangle$ or $\langle 110\rangle$ GB migration modes ${ }^{924}$ for the $\Sigma 13(320)[001]$ and $\Sigma 17(410)$ GB. The displacements (displayed by an arrow in Fig. 5b) in the [320] direction of 3 atoms (blue circled in Fig. $5 \mathrm{~b}$ ) are mainly responsible of the disconnection motion. A dashed line, a guide to the eyes features the position of the structural units after the motion (to the left) of the disconnection of Fig. 5b). No disconnection kink along the [001] direction has been observed during a $\langle 010\rangle$ GB migration mode for all the investigated shear displacement and normal stress. The absence of disconnection kink is related to the limited size of the simulation cell in the [001] direction. Due to the high number of atoms in the cell and the high number of images necessary to get the convergence of NEB simulations, we did not try to increase the size of the cell in the [001] direction in order to evidence disconnection kinks 24 .

The following section is devoted to the results and discussion on the activation energies dependence on the shear displacements and normal stress.

\section{ACTIVATION ENERGIES}

Fig. 6 reports the activation energies as a function of the normal stress $\sigma_{N}$ and of the shear displacement $d$ : both a 3D plot and top view are reported. Fig. 6 contains 226 points, each corresponding to a NEB calculation involving from 50 to 100 images: the computational effort in order to produce Fig. 6 is thus significant. Unfortunately, despite these numerous efforts, we did not succeed to get the convergence of every NEB calculations, which explains that a few points are missing on the graph: the number of points is however sufficiently large to get an overall understanding of the occurrence of the $\langle 110\rangle$ and $\langle 010\rangle$ GB migration modes. In addition, we have limited the calculation of the activation energies of the modes $\langle 110\rangle(\langle 010\rangle)$ for normal stresses $\sigma_{N}$ for which the $\langle 110\rangle(\langle 010\rangle)$ mode is observed at $0 \mathrm{~K}$ i.e. for $\sigma_{N}<4.5 \mathrm{GPa}\left(\sigma_{N}>4.5 \mathrm{GPa}\right)$. The activation energies for any normal stress and shear displacement can in principle be evaluated using the NEB method providing the generation of the judicious initial and final configurations. These latter configurations can be prepared from elastic deformations of GB configurations obtained in alternatives conditions: such a work has previously been performed for the $\langle 110\rangle$ and $\langle 100\rangle$ modes of a $\mathrm{Cu}$ $\Sigma 17(410)$ GB 24 but has not been reproduced here due to the very high computational cost that it would imply.

The activation energies of the $\langle 010\rangle$ mode for $\left(\sigma_{N}\right\rangle$ $4.5 \mathrm{GPa}$ ) are generally much larger, typically one order of magnitude, than the $\langle 110\rangle$ one $\left(\sigma_{N}<4.5 \mathrm{GPa}\right)$. This difference is in quantitative agreement with the ratio of elastic energies carried by the $\langle 010\rangle$ and $\langle 110\rangle$ mode disconnections: indeed, the $\langle 010\rangle$ disconnection Burgers vector norm is three times larger than the $\langle 110\rangle$ one and the elastic energy scales as the square of the Burgers vector square $^{1}$.

For both modes, as expected and already observed 8 [24, GB migration activation energies decrease as a function of the shear displacement. However, the decrease is much more pronounced for the $\langle 010\rangle$ GB migration modes than for the $\langle 110\rangle$ one: for the $\langle 010\rangle(\langle 110\rangle)$ modes activation energies roughly changes from around $25-30 \mathrm{eV}(2-$ $3 \mathrm{eV}$ )for null value of the shear displacements to null activation energies for value of the shear displacements corresponding to about $1 \mathrm{~nm}$ (varying from 0.2 to $1 \mathrm{~nm}$ depending on the normal stress). The difference of activation energies decrease depending on the mode is in agreement with the larger Burgers vector of the $\langle 010\rangle$ 


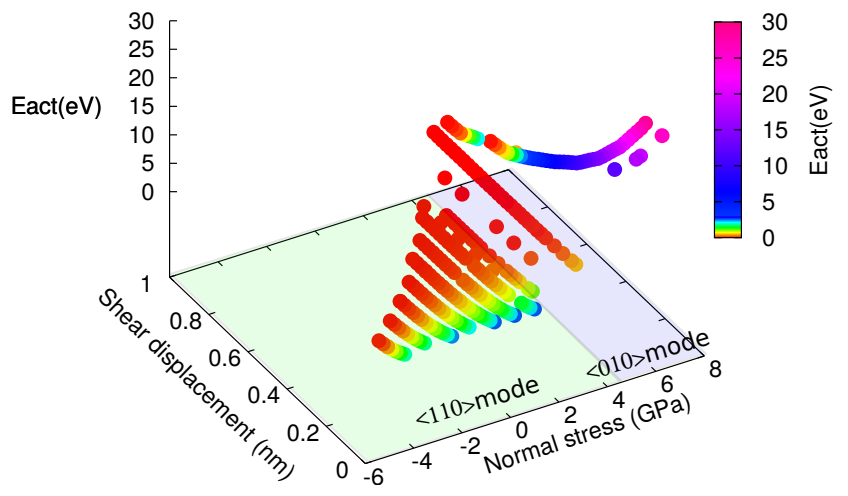

a)

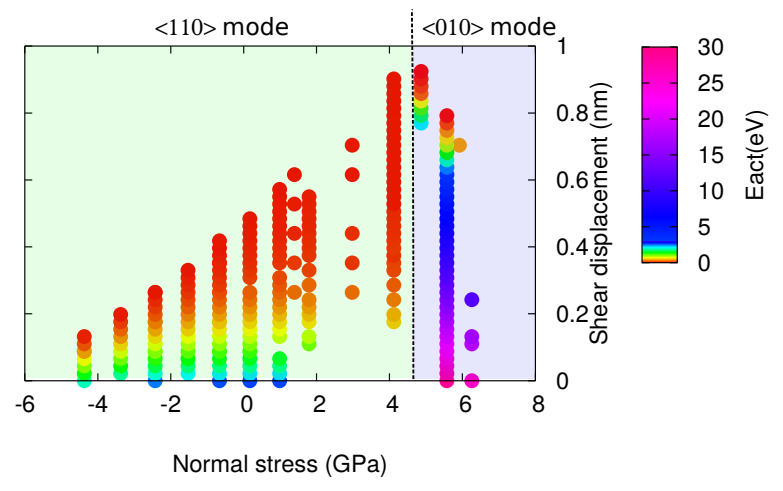

b)

FIG. 6. (color online) Activation energies of the GB migration as a function of the normal stress $\sigma_{N}$ and of the shear displacement $d$. a) 3D plot and b) Top view. The operating regions of the $\rangle 110\left\langle\left(\sigma_{N}<4.5 \mathrm{GPa}\right)\right.$ and $\rangle 010\left\langle\left(\sigma_{N}>4.5\right.\right.$ $\mathrm{GPa})$ modes are highlighted.

mode disconnection than the $\langle 110\rangle$ one. Indeed, the GB migration activation energy is expected to roughly decrease as the product of the Burgers vector norm times the shear stress (corresponding to the mechanical work for the shear displacement). Hence, though the activation energies of the $\langle 010\rangle$ are generally much larger than the $\langle 110\rangle$ one, the $\langle 010\rangle$ GB migration mode may become observable at high shear displacement since its activation energy may cancel for smaller shear displacement $d$ than for the $\langle 110\rangle$ mode. This situation precisely occurs for $\sigma_{N}>4.5 \mathrm{GPa}$ and is similar to the competition between the $\langle 110\rangle$ and $\langle 100\rangle$ modes observed in a $\mathrm{Cu} \Sigma 17(410)$ $\mathrm{GB}^{24}$.

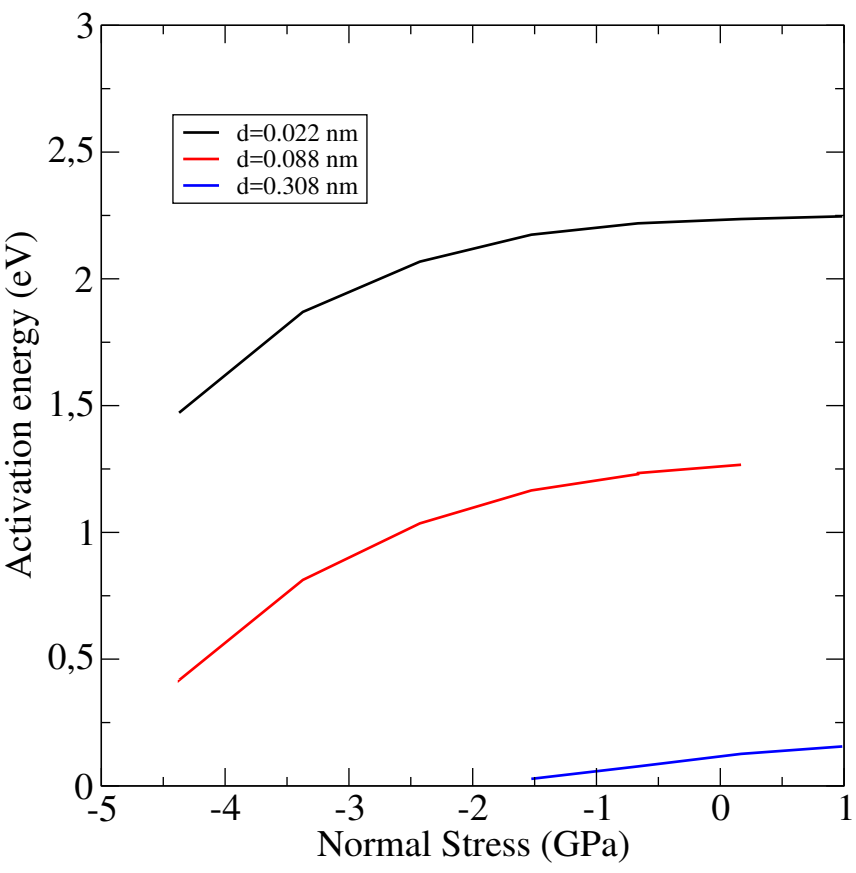

FIG. 7. (color online) Activation energies of the GB migration for the $\langle 110\rangle$ mode as a function of the normal stress $\sigma_{N}$ for three values of the shear displacement $d$ reported in the legend.

\section{DISCUSSION}

The tensile normal stress $\sigma_{N}>4.5 \mathrm{GPa}$ necessary to observe the $\langle 110\rangle$ mode of the $\Sigma 13(320)[001]$ GB at zero temperature is very high: indeed, it is close to the tensile normal stress necessary to initiate fracture in the GB plane. We thus think that such tensile normal stresses are not compatible with an experimental set up. In addition, in our model, for non-extreme shear displacement values, the activation energies of the $\langle 010\rangle$ mode are typically one order of magnitude higher than the $\langle 110\rangle$ mode ones. Hence, assuming our results are transposable to experiments, we would expect that the actual observation of the $\langle 010\rangle$ mode $\Sigma 13(320)[001]$ GB migration will be quasiimpossible at finite temperature. We thus conclude that, within a reasonable range of experimental conditions, the observation of the $\langle 010\rangle$ mode of the $\Sigma 13(320)[001] \mathrm{GB}$ in real crystals appears very unlikely.

However, the present study shows that the effect of a normal stress on the GB migration mechanism is effective. This conclusions is reinforced by similar results in dislocation plasticity: experiments have demonstrated that a high hydrostatic pressure can allow a plastic deformation of materials at temperature lower than the ductile to brittle transition: these results were interpreted by the reduction of activation energies of thermal activated processes such as dislocation climb $\stackrel{37}{\text { or glide }}{ }^{38}$. Therefore, we reasonably expect that the migration of other GBs (especially other orientations) in different materials under a normal load will lead to cases for which the 
$\langle 010\rangle$ mode operation will be favorable. In addition, one can also expect to uncover other unobserved or new GB migration mechanisms from such studies.

Finally, another interesting information of Fig. 6 concerns the $\langle 110\rangle$ mode. Fig. 2 shows that for this mode, the yield shear stress increases with the normal stress, suggesting that a GB would migrate more easily when submitted to a compressive stress i.e. for a smaller applied yield shear stress than one submitted to a tensile stress at $0 \mathrm{~K}$. This picture is actually confusing since it is altered at finite temperature. Fig. 7 reports the activation energies as a function of the normal stress for three different values of the slab displacement. For a given shear displacement $d$, the GB migration activation energy barely depends on the normal stress as soon as the normal stress is above a critical value $\sigma_{c}$ depending on the shear displacement:from fig. 7, this critical value roughly corresponds to $\sigma_{c}=-1.5 \mathrm{GPa}$ for $d=0.022 \mathrm{~nm}$. Hence, at finite temperature, for normal stresses higher than this critical value, the application of a normal stress will have not almost effect on the GB mobility. This result agrees with the previous simulations on the $\Sigma 11(113)$ $\mathrm{Cu}$ GB showing that the GB migration at $300 \mathrm{~K}$ is not significantly affected by the presence of a normal stress 26 . Hence, the effects of the normal stress on GB migration can qualitatively differ at null or finite temperature depending on the precise conditions under which the GB migration is studied.

\section{CONCLUSION}

This numerical study reports on the influence of a normal stress on the shear coupled GB migration mechanism. We have shown that at zero temperature, the application of a normal stress significantly affects the values of the yield shear stress. We have shown that a normal stress can even induce a modification of the GB migration mechanism. We have reported simulations conditions allowing the operation of the $\langle 010\rangle$ GB migration mode. To our knowledge, this mode, theoretically predicted years ago has never been observed in experiments nor numerical studies. We have shown that the GB migration occurs through the nucleation of two disconnections that were characterized. Finally, we have calculated the activation energies for disconnection nucleation.

We believe that the present study complements our previous studies924: indeed, our studies have shown that a GB can migrate following several mechanisms, each of them characterized by a given disconnection and activation energy. All of these mechanisms compete with each other. The activation energies depend on the conditions under which the migration is induced: with our previous and present studies, we have explored the effects of temperature (see also Ref ${ }^{[12}$ ), shear stress (or shear displacement) and now of the application of a normal stress.

The present study opens a vast field of possible perspectives. We report below two possible directions of investigations. First, this work has been intentionally limited to conditions that preserves the GB structures. But as mentioned in Sect. If the study of the GB migration with free boundary conditions is also relevant and would certainly also bring additional interesting information on the shear-coupled GB migration: it will possibly reveal additional potential GB mechanism.

Second, a more systematic study of the migration of various GBs (including asymmetric GB) under various conditions will likely evidence new GB migration mechanisms. The knowledge and the characterization of all these possible mechanisms is a key ingredient in order to understand and improve the plastic behavior of nano-crystalline materials under stress.
* nicolas.combe@cemes.fr

1 J. P. Hirth and J. Lothe, Theory of dislocations (Krieger Publishing Company, Malabar, Florida, 1992).

2 E. Hall, Proc. Phys. Soc. B 64, 747 (1951)

3 N. J. Petch, J. Iron Steel Inst. 174, 25 (1953).

${ }^{4}$ T. Gorkaya, D. A. Molodov, and G. Gottstein, Acta mat. 57, $5396(2009)$

5 G. Gottstein, D. Molodov, L. Shvindlerman, D. Srolovitz, and M. Winning, Curr. Opin. Solid State Mater. Sci. 5, 9 (2001)

${ }^{6}$ M. Legros, D. Gianola, and K. Hemker, Acta Mat. 56, $3380(2008)$

A. Rajabzadeh, M. Legros, N. Combe, F. Mompiou, and D. Molodov, Phil. Mag. 93, 1299 (2013)

8 A. Rajabzadeh, F. Mompiou, S. Lartigue-Korinek, N. Combe, M. Legros, and D. Molodov, Acta Mat. 77, $223(2014)$.
9 A. Rajabzadeh, F. Mompiou, M. Legros, and N. Combe, Phys. Rev. Lett. 110, 265507 (2013)

10 M. A. Meyers, A. Mishra, and D. J. Benson, Prog. Mat. Sci. 51, 427 (2006).

11 J. Schäfer and K. Albe, Acta Mat. 60, 6076 (2012).

12 J. W. Cahn, Y. Mishin, and A. Suzuki, Acta Mat. 54, 4953 (2006)

${ }^{13}$ D. Caillard, F. Mompiou, and M. Legros, Acta Mat. 57, 2390 (2009).

${ }^{14}$ F. Mompiou, D. Caillard, and M. Legros, Acta Mat. 57, 2198 (2009).

is Y. Mishin, A. Suzuki, B. P. Uberuaga, and A. F. Voter, Phys. Rev. B 75, 224101 (2007).

16 D. Olmsted, E. A. Holm, and S. Foiles, Acta Mat. 57, 3704 (2009)

If E. R. Homer, S. M. Foiles, E. A. Holm, and D. L. Olmsted, Acta Mat. 61, 1048 (2013). 
18 C. P. Race, J. von Pezold, and J. Neugebauer, Phys. Rev. B 89, 214110 (2014)

I9 E. R. Homer, S. Patala, and J. L. Priedeman, Nature 5, $15476(2015)$

${ }^{20}$ L. Wan and S. Wang, Phys. Rev. B 82, 214112 (2010)

21 J. Hirth, J. Phys. Chem. Solids 55, 985 (1994).

22 J. Hirth and R. Pond, Acta Mat. 44, 4749 (1996).

${ }^{23}$ H. Khater, A. Serra, R. Pond, and J. Hirth, Acta Mat. 60, 2007 (2012)

${ }^{24}$ N. Combe, F. Mompiou, and M. Legros, Phys. Rev. B 93, 024109 (2016).

25 D. A. Molodov, T. Gorkaya, and G. Gottstein, J. Mater. Sci. 46, 4318 (2011)

${ }_{20}$ D. H. Warner and J. F. Molinari, Modelling Simul. Mater. Sci. Eng. 16, 075007 (2008).

${ }^{2}$ J. W. Cahn, Y. Mishin, and A. Suzuki, Phil. Mag, 86, 3965 (2006)

28 S. J. Plimpton, J. Comp. Phys. 117, 1 (1995)

29 Y. Mishin, D. Farkas, M. J. Mehl, and D. A. Papaconstantopoulos, Phys. Rev. B 59, 3393 (1999)
30 For an isotropic material, using the linear elasticity theory, the in-plane stress would be the product of the normal strain by the second Lamé coefficient ${ }^{33}$.

31 A. P. Thompson, S. J. Plimpton, and W. Mattson, J. Chem. Phys. 131, 154107 (2009).

32 T. Frolov, D. L. Olmsted, M. Asta, and Y. Mishin, Nature Com. 4, 1899 (2013)

${ }^{33}$ L. D. Landau and E. M. Lifshitz, Theory of elasticity (Pergamon Press, Oxford, 1986).

${ }^{34}$ D. A. Molodov, T. Gorkaya, and G. Gottstein, Scripta Materialia 65, 990 (2011)

35 G. Henkelman, B. P. Uberuaga, and H. Jonsson, J. Chem. Phys. 113, 9901 (2000)

36 Z. Trautt and Y. Mishin, Acta Mat. 60, 2407 (2012)

37 F. Mompiou and D. Caillard, Acta Mat. 52, 3613 (2004)

38 J. Rabier, P. Cordier, J. Demenet, and H. Garem, Mater. Sci. Eng. A 309-310, 74 (2001) 